\title{
Influence of decoupling effect on stellar wind variability
}

\author{
Viktor Votruba ${ }^{1,2}$, Klára Šejnová ${ }^{2}$, Pavel Koubský ${ }^{1}$ \\ and Daniela Korčáková ${ }^{1}$ \\ ${ }^{1}$ Stellar Department, Astronomical Institute AV ČR, v.v.i., Fričova 298, \\ Ondřejov 25165, Czech Republic; email: votruba@physics.muni.cz \\ ${ }^{2}$ Institute of Theoretical Physics and Astrophysics, Masaryk University, Faculty of Natural \\ Science, Kotlářská 2, Brno 61137, Czech Republic; email: klarka@physics.muni.cz
}

\begin{abstract}
A detailed investigation of momentum transfer in radiatively driven stellar winds shows that for the thin wind case ion decoupling may occur. The decoupling of absorbing ions significantly affects dynamics of the wind. We analysed such effects using our hydrodynamic code and predicted spectral changes with the help of the SHELLSPEC code.
\end{abstract}

Keywords. stars: winds, outflows, stars: oscillations (including pulsations)

\section{Introduction}

The assumption of a one-component flow used by the CAK theory is acceptable for most cases of stellar winds from $\mathrm{O}$ and hot B stars. But for stars with a low-density wind it is necessary to use a detailed description of the momentum transfer. As was shown first by Springmann \& Pauldrach (1992), the gas and radiation field can decouple in the flow generated by a low-density radiatively driven wind. This is because in the low-density wind, Coulombic collisions are not effective and cannot support momentum transfer from absorbing ions to the passive plasma. As a result, the wind decouples at a certain point.

A very interesting result from the analysis of the decoupling effect is the generation of pulsating shells, which was first suggested by Porter \& Skouza (1999). In the case when winds decouple at the point where the local velocity of the flow is still smaller than the escape velocity, the passive plasma is still gravitationally bound to the star. This implies that matter is decelerated and falls back down to the star. The interaction with an outflow from the star leads to the pulsating shells.

\section{Numerical Simulation}

We use the hydrodynamic code developed by Feldmeier (1995) for the simulation of pulsating shells, which uses an Euler scheme with Van Leer flux splitting. To model the decoupling effect we terminated the radiative force at a distance $r_{d}$, where the flow was still gravitationally bound to the star. It was also necessary to change the outflow boundary condition and suppress material flow from the outer boundary. The chosen stellar parameters can be found in Table 1 . The time of the simulation was roughly $\sim 2 \mathrm{~d}$.

Table 1. Stellar parameters of the model

\begin{tabular}{|c|c|c|c|c|}
\hline$M\left[\mathrm{M}_{\mathrm{sol}}\right]$ & $R_{s}\left[\mathrm{R}_{\odot}\right]$ & $T[\mathrm{~K}]$ & $\dot{M}\left[\mathrm{M}_{\odot} \cdot \mathrm{y}^{-1}\right]$ & $r_{\mathrm{d}}\left[\mathrm{R}_{s}\right]$ \\
\hline 13.0 & 7.0 & 19300 & $1.210^{-9}$ & 1.25 \\
\hline
\end{tabular}



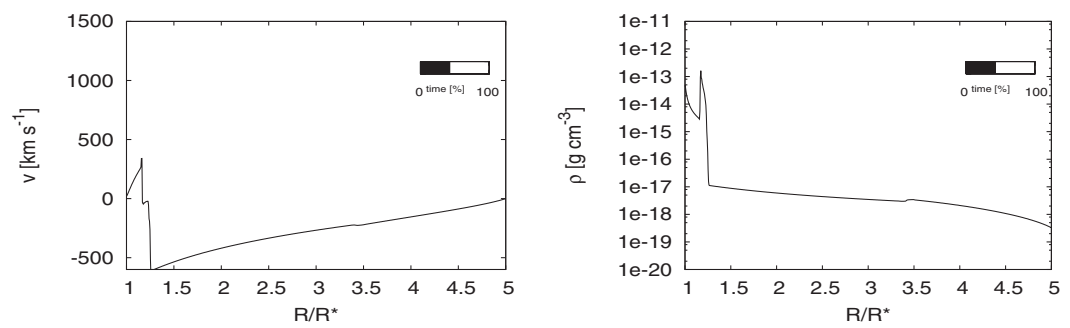

Figure 1. Snapshot of the pulsating shell for a model star. Left panel: Velocity distribution in the flow. Right panel: Density distribution in the flow. Spherical shells are clearly visible as enhanced density regions in the plot.

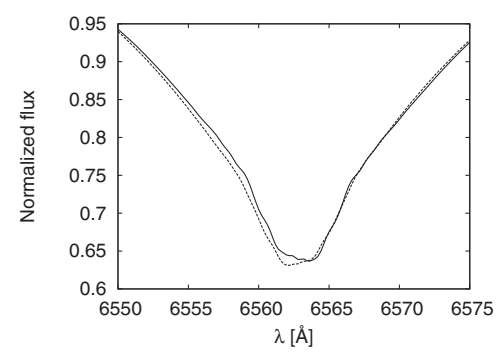

Figure 2. $\mathrm{H}_{\alpha}$ line profiles. Two lines mark two different groups of input parameters. The solid line corresponds to the infalling shell with velocity $\sim-500 \mathrm{~km} . \mathrm{s}^{-1}$, density $\sim 1.10^{-14} \mathrm{~g} . \mathrm{cm}^{-3}$ and thickness $\sim 7 \mathrm{R}_{\text {sol }}$. The dashed line corresponds to outflow velocity $\sim 500 \mathrm{~km} . \mathrm{s}^{-1}$, density $\sim 1 .^{-15}$ g.cm ${ }^{-3}$ and thickness $\sim 12 \mathrm{R}_{\text {sol }}$.

\section{Synthetic spectral changes}

The key question is if there is an observable signature of the decoupling effect. The most obvious effect of decoupling is that the observed terminal velocity will be much lower than predicted from CAK theory. This effect can be also detected directly from the stellar spectra. As was first predicted by Porter \& Skouza (1999), pulsating shells may provide a varying component to the hydrogen lines. Under the assumption of an optically thin shell we used the SHELLSPEC code for the estimation of spectral line variations.

SHELLSPEC, developed by Budaj \& Richards (2004), is designed to solve simple radiative transfer along the line of sight in moving media. The output of the code is: synthetic spectra, light curve or trailing spectrogram. Since generated shells pulsate roughly periodically, we analysed 2 different cases: when the outflowing shell is moving away from the star and when the shell is falling back toward the star. For both stages we derived the geometry and dynamical properties of the problem from our numerical simulations and applied the SHELLSPEC code. The resulting spectral variations are shown in Fig. 2.

\section{Acknowledgements}

This research was supported by grants 205/09/P476 (GA ČR) and 205/08/H005 (GA ČR). The Astronomical Institute Ondřejov is supported by project AV0Z10030501.

\section{References}

Budaj, J. \& Richards, M. T. 2004, Contributions of the Astronomical Observatory Skalnate Pleso 34,167

Feldmeier, A. 1995, A\&\&A, 299, 523

Porter, J. M. \& Skouza, B. A. 1999, A\&SA, 344, 205

Springmann, U. W. E. \& Pauldrach, A. W. A. 1992, A\& A, 262, 515 